\title{
Pseudopotential SCF-MO studies of hypervalent compounds. IV. Structure, vibrational assignments, and intramolecular forces in $\mathrm{IF}_{7}$
}

\author{
Lawrence S. Bartell, Michael J. Rothman, and Angelo Gavezzottia) \\ Department of Chemistry, University of Michigan, Ann Arbor, Michigan 48109 \\ (Received 18 December 1981; accepted 8 January 1982)
}

Ewig's pseudopotential procedure, applied with a small basis set, yielded a minimum energy structure with $D_{s_{h}}$ symmetry instead of the deformed pseudorotating structure proposed to account for electron diffraction and electric beam deflection experiments. Nevertheless, calculated restoring forces for $e_{2}^{\prime \prime}$ displacements were so feeble that a higher quality calculation making the postulated $e_{2}^{\prime \prime}$ deformation spontaneous is not at all precluded. Otherwise, calculated molecular characteristics of $\mathbf{I F}_{7}$ closely resembled those deduced from the diffraction and beam experiments, from extended Hückel calculations, and from the simple valence shell electron pair repulsion theory in a repelling points-on-a-sphere (POS) variant. Axial bonds were shorter by $0.05 \AA$ than equatorial bonds, and calculated mean bond lengths exceeded observed ones by only $0.03 \AA$. Furthermore, the calculated anharmonic coupling of $e_{1}^{\prime}$ polar displacements with large-amplitude $e_{2}^{*}$ puckering vibrations closely reproduced the coupling inferred from experiment. This favorable correspondance between theory and interpretation of experiment was found not to extend to vibrational assignments, however. Instead, the pseudopotential calculations were in suprisingly close agreement with the simple POS and EHT models predicting that equatorial in-plane bends are far stiffer, due to the close lateral contacts, than the other bending modes. None of the six published vibrational assignments had been interpreted on this basis. An alternative assignment was found which is reasonably consistent with both the calculated force field and the observed infrared and Raman spectra. A similar assignment seems appropriate for $\mathrm{ReF}_{\gamma}$. Calculations with and without $d$ orbitals on iodine furnished evidence that the role of $d$ orbitals in hypervalent compounds is less important than it is often considered to be. Neither the second-order Jahn-Teller softening of the $e_{2}^{\prime \prime}$ force constants nor the covalent binding of seven fluorines to iodine was found to depend crucially upon valence $d$ orbitals.

\section{INTRODUCTION}

Iodine heptafluoride is an interesting quasispherical molecule that has only grudgingly revealed its internal structure. Drawing its neon-like sheath of fluorines tightly around itself, it is highly volatile, though heavy (subliming at $4{ }^{\circ} \mathrm{C}$ ), ${ }^{1}$ much more inert to hydrolysis than other hypervalent halogen fluorides, ${ }^{2}$ and difficult to characterize by standard physical procedures. Because of its shape and comparatively weak cohesive forces, it disorders easily when crystallizing, thereby hampering $x$-ray structure determinations. The several crystal structure analyses performed upon it led to somewhat contradictory results. ${ }^{3-8}$ Yet, as one of only two known seven-coordinate binary compounds stable in the vapor phase, (the other being $R e F_{\eta}$ ) the molecule is of potentially far greater value than most others as an illustration of how covalent bonds interact with each other. To show this, it is helpful to introduce one of the simplest and most widely used pictures to account for directed valence, the Sidgwick-Powell-Gillespie-Nyholm "valence-shell-electron-pair-repulsion" (VSEPR) theory. ${ }^{\text {, }}$, It makes definite and, for the most part, surprisingly satisfactory predictions of the structures ${ }^{9,10}$ and force field characteristics ${ }^{11-13}$ of molecules consisting of a central atom (in the main group) with six or fewer electron pairs in its valence shell. In the case of seven pairs (e.g., $\mathrm{XeF}_{6}$ or $\mathrm{IF}_{7}$ ), however, purely geometric considerations of "electron repulsion" are no longer sufficient and predicted structures range from $D_{5 h}$

\footnotetext{
${ }^{a}$ Permanent address: Instituto di Chimica Fisica, Università di Milano, Milan, Italy
}

through a series of intermediate configurations on to $C_{3 v}$ for "hard repulsions." 14,15 Therefore, an accurate structure determination would presumably yield not merely another example that bonds tend to avoid each other, but it would also reveal the "force law" associated with this avoidance. If this seems an excessively naive and simplistic hope, we point out that the present investigation strongly reinforces the virtue and utility of this mode of interpretation.

The best structural information available indicates that $\mathrm{IF}_{7}$ spends most of its time distorted by a weakly polar $\left(e_{2}^{\prime \prime}+e_{1}^{\prime}\right)$ defor mation from a $D_{5 h}$ configuration. ${ }^{16,17}$ The molecule is believed to be undergoing a dynamic ring-puckering pseudorotation. Structural inferences were drawn mainly from subtle features of a vaporphase electron diffraction study, ${ }^{16}$ and the polar character was corroborated by a molecular beam investiga tion. ${ }^{18}$ Although the main features deduced by electron diffraction analysis are almost certainly correct, analyses were impeded somewhat by intramolecular multiple scattering ${ }^{19}$ of incident electrons by the heavy iodine atom. At the time of analysis, no satisfactory theory for this phenomenon had yet been worked out. Despite its weak polarity, the molecule has displayed no pure rotational spectrum. It has continued to resist attempts to establish its symmetry and force field by vibrational spectroscopy. ${ }^{20-29}$ Until the electron dif fraction study, vibrational spectra had been interpreted in terms of a simple $D_{5 n}$ structure. ${ }^{20-25}$ Even though subsequent infrared and Raman analyse ${ }^{26-29}$ have been considered to be compatible with the distorted structure suggested by the diffraction experiment, the vibrational 
assignments are far from resolved. Each new study has suggested a different assignment. Furthermore, evidence, which we shall develop in the following, suggests that all of the published assignments are incorrect.

For the above reasons it seemed worthwhile to bring to bear an alternative method in an attempt to find the key to this interesting but elusive molecule. One promising approach is an $a b$ initio pseudopotential SCF-MO method developed by Ewig and Van Wazer. ${ }^{30}$ In previous papers in this series, ${ }^{31-34}$ it was applied in single determinant for $m$, to nonrelativistic calculations of the structures and force fields of the molecules $F_{4}, X_{2} F_{2}, X_{4} F_{4}$, $\mathrm{XeF}_{5}^{*}, \mathrm{XeF}_{6}, \mathrm{Te}_{4}^{2+}, \mathrm{I}_{2}, \mathrm{IF}, \mathrm{IF}_{3}$, and $\mathrm{IF}_{5}$. Results, where comparisons could be made, were comparable to those of all-electron SCF calculations with similar basis sets. Although calculated bond lengths, bond angles, and force constants deviated from experimental values in systematic ways, the pattern of calculated molecular properties proved of value in interpreting existing observations. In the case of $\mathrm{IF}_{7}$, it was unrealistic to expect to be able to compute the structure and vibrational frequencies with sufficient absolute accuracy to give direct answers to the unresolved problems. On the other hand, it was likely that useful clues would be found which would clarify the situation considerably when prior experience with related molecules was taken into account. Adding to our optimism that the properties of $\mathrm{IF}_{7}$ were decipherable were strong hints about the force field provided by previous computations based on two very simple models, the VSEPR concept of repeling points -on-asphere (POS) ${ }^{11-16}$ and extended Hückel theory (EHT). ${ }^{11,35}$ How these contribute to the analysis will be described briefly in the following section.

\section{PROCEDURE}

\section{A. Pseudopotential calculations}

The computational method adopted in the present research is described in detail in Refs. 30-32. It is $a b$ initio in its entirety. Basis sets for fluorine $e^{32}$ and iodine ${ }^{34}$ were constructed from multizeta valence, singlezeta core orbitals of Clementi et al ${ }^{36,37}$ and contracted to a minimal basis set superior to single zeta STO's. Force constants were computed as outlined in previous papers in this set. ${ }^{31-33}$ Because a gradient procedure had not been incorporated into the computer code to facilitate energy minimization with respect to structure parameters, the minimum energy $D_{5 h}$ structures and $a_{1}^{\prime}$ force constants were found as follows.

The experimental ${ }^{16}$ mean bond lengths $r_{0 Q}^{0}=1.858 \AA$ and $r_{\mathrm{ax}}^{0}=1.786$ were adopted as reference values. Total molecular energies for this structure and other $D_{5 h}$ structures with bond lengths $\left(r_{\text {og }}^{0}+u, r_{a x}^{0}+v\right)$ were calculated at displacements $(u, v)$ in $\AA$ of $(-0.1,0),(0.1,0)$, $(0,0.08),(0,0.16)$, and $(0.1323,0.1323)$. For displace ments of this magnitude the Morse anharmonicity is not negligible. In order to allow for it without going to the appreciable expense of additional pseudopotential computations, we adopted the Morse parameter $a \approx 1.6 \AA^{-1}$ found in related computations on xenon fluorides ${ }^{23}$ and fitted the above points exactly with the polynomial

$$
\begin{aligned}
2 V(u, v)= & 2 V_{0}+k_{\mathrm{ea}}\left[\left(u-u_{0}\right)^{2}-a\left(u-u_{0}\right)^{3}\right] \\
& +k_{\mathrm{ard}}\left[\left(v-v_{0}\right)^{2}-a\left(v-v_{0}\right)^{3}\right]+2 k_{a \mathrm{e}} u v .
\end{aligned}
$$

From this polynomial can be found $u_{m}, v_{m}$, the displacement coordinates at minimum energy as well as the symmetry force constants $F_{11}, F_{22}$, and $F_{12}$ at either the experimental or theoretical structures.

\section{B. Points-on-a-sphere force constants}

For reasons that will become apparent, it is useful to be able to calculate the profile of bending force constants corresponding to the simple model of seven repeling points on a sphere, the POS model. In the VSEPR model of Gillespie ${ }^{10}$ these repulsions are envisioned as acting, presumably by the operation of the exclusion principle, between electron pairs in the IF bonds of $\mathrm{IF}_{7}$. In a Urey-Bradley model they would, alternatively, be consider ed to be atom-atom nonbonded repulsions. Whatever the source of repulsion, we shall assume for this section that the repulsions correspond to pairwise additive potential energy terms $V\left(r_{i j}\right)$ acting between points $i$ and $j$ separated by $r_{1 j}$ and constrained to move on a surface of radius $R$. A reference structure of $D_{5 k}$ symmetry is adopted. In prior work the potential terms $V\left(r_{i j}\right)$ have often been expressed in explicit form such as $K r_{i j}^{-n}$ or, less often, as $C \exp \left(-\alpha r_{i j}\right)$. For the present we leave them general. By expanding the potential function into a Taylor series we derive the following relations for quadratic bending force constants relative to a $D_{5 h}$ reference structure:

$$
\begin{aligned}
F_{44}\left(a_{2}^{\prime \prime}\right)= & F\left(90^{\circ}\right)-\frac{1}{2} R \sin 36^{\circ}(\partial V / \partial r)_{72^{\circ}-} \\
& -\frac{1}{2} R \sin 72^{\circ}(\partial V / \partial r)_{144^{\circ}}, \\
F_{66}\left(e_{1}^{\prime}\right)= & \mathcal{F}\left(72^{\circ}\right)+\frac{1}{4} R^{2}\left(\partial^{2} V / \partial r^{2}\right)_{144^{\circ}} \\
& -2 R \cos ^{2} 144^{\circ} \sin 72^{\circ}(\partial V / \partial r)_{144^{\circ}}, \\
F_{67}\left(e_{1}^{\prime}\right)= & \left(R / 2 \sin 36^{\circ}\right)(\partial V / \partial r)_{90^{\circ}}, \\
F_{77}\left(e_{1}^{\prime}\right)= & F\left(90^{\circ}\right)-0.4 R(\partial V / \partial r)_{180^{\circ}}, \\
F_{99}\left(e_{2}^{\prime}\right)= & F\left(72^{\circ}\right)+\frac{R^{2} \cos ^{2} 72^{\circ}}{4 \cos ^{2} 144^{\circ}}\left(\frac{\partial V}{\partial r}\right)_{144^{\circ}} \\
+ & \frac{1}{4} R \tan 144^{\circ}(\partial V / \partial r)_{144^{\circ}}, \\
F_{10.10}\left(e_{1}^{\prime \prime}\right)= & F\left(90^{\circ}\right), \\
F_{11,11}\left(e_{2}^{\prime \prime}\right)= & F\left(90^{\circ}\right)+R \sin 72^{\circ}(\partial V / \partial r)_{72^{\circ}} \\
& -R \sin 144^{\circ}(\partial V / \partial r)_{144^{\circ}},
\end{aligned}
$$

where

$$
\mathcal{F}(\theta)=R^{2} \cos ^{2}(\theta / 2)\left(\partial^{2} V / \partial r^{2}\right)_{\theta}-\frac{1}{2} R \sin (\theta / 2)(\partial V / \partial r)_{\theta},
$$

in which $\theta$ represents one of the interbond angles in a pentagonal bipyramid.

\section{Points-on-a-sphere normal coordinate calculations}

The intended use of the equations in the preceding section is to permit a direct comparison between the force field implied by the simple POS model and calculated fields with a more conspicuous quantum origin. As a rough guide to assignments, how ever, we car ried out normal coordinate calculations ${ }^{38}$ using an al- 
TABLE I. Preliminary points-on-asphere potential function for normal coordinate analysis of $\mathrm{IF}_{70^{\circ}}{ }^{2}$ Relaxed sphere radius $1.858 \AA$.

\begin{tabular}{lcc}
\hline \hline $\begin{array}{c}\text { Bond stretching: } \\
\text { Bond }\end{array}$ & $V(r)=\frac{1}{2} R\left(r-r^{0}\right)^{2}$ \\
& $k$ & $r^{0}$ \\
\hline Axial & 4.2 & 1.584 \\
Equatorial & 3.4 & 1.471
\end{tabular}

Atom-atom repulsion: $V(r)=4.66 / r^{3.5}$

${ }^{2}$ Energies in mdyn $\AA$, distances in $\AA$; for calculating bending frequencies alone, $K$ can be made enormous and $r^{0}$ set at $1.858 \AA$.

ternative representation of a modified POS field. This field, in which valence force constants were never explicitly calculated, was formulated in order to cope with the following problem. As can be seen from the expression for $F_{11,11}$ above, the force constant for $e_{2}^{\prime \prime}$ ring puckering deformations becomes negative when the POS repulsions exceed a certain hardness (e.g., when $n$ exceeds 2 for $\left.V_{i j}=K / r_{i j}^{n}\right)$. Under such a condition an $e_{2}^{\prime \prime}$ deformation becomes spontaneous and it, in turn, induces an $e_{1}^{\prime}$ axial bend via a cubic potential constant coupling the modes. Not only is such a possibility mathematically possible but it seems to be encountered in reality in the $\mathrm{IF}_{7}$ molecule, according to evidence mentioned in the Introduction. In the case of such a fluxional, freely pseudorotating molecule it is not a trivial matter to express the structure and force field on a valence force constant basis. On the other hand, it is comparatively simple to invoke an atom-atom

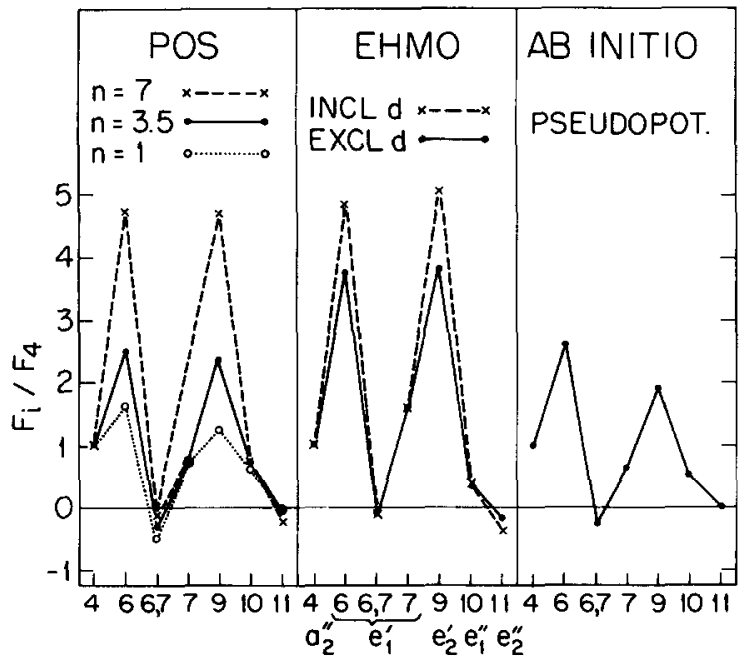

FIG. 1. Bending quadratic force constant profile calculated for $\mathrm{IF}_{7}$ as a function of mode number by various approaches. Interaction constant $F_{6,7}$ is designated as 6,7 . (a) Repelling points-on-a-sphere results for three values of repulsion hardness parameter $n$. A value of 3.5 accounted for observed structure characteristics, Ref. 10. (b) Extended Hückel results including and excluding $d$ orbitals. (c) Effective-potential results described in text. (or bond-bond) repulsion of the desired magnitude and hardness to fit, approximately, both the observed structural deformation from $D_{5 h}$ and the bending frequencies. In addition, the bonds can be given Hookean force constants so that the stretch modes can be calculated, as well. If one had constructed such a field with identical reference bond lengths, the equatorial bonds would have stretched further under the atom-atom repulsions than the axial, much as implied by experiment. We chose arbitrarily instead, however, to assign differ ent reference lengths to axial and equatorial bonds so that the final, relaxed structure gave equal (1. $858 \AA$ ) bond lengths with atoms residing on the POS surface. This was for sake of comparison with prior calculations carried out with the POS model. ${ }^{14,16,39}$ Had unequal bond lengths been retained, the hardness parameter " $n$ " to give the observed angle deformations would have been different. Parameters for the potential function, chosen for the present purposes with no attempt to optimize them, are listed in Table I.

One virtue of such a normal coordinate calculation based on a deformed structure is that it indicates the extent to which the modes depart in form from those expected for a $D_{5 h}$ structure. Such allowed mixing of modes is of concern in the plausibility of a revised assignment.

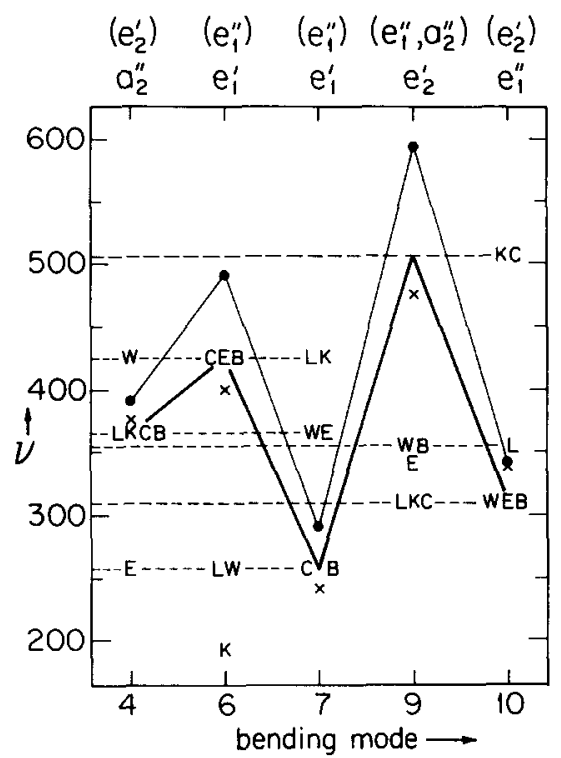

FIG. 2. Bending frequency assignments by various authors (capital letters, see below). Assignments suggested by present calculations are connected by the heavy line. Frequencies calculated by the pseudopotential method are connected by the light line. Dashed horizontal lines correspond to reported experimental frequencies. Crosses correspond to frequencies calculated by the POS force field of Table I. Symmetry designations above the figure represent dominant displacements (not in parentheses) and important secondary vibrational displacements (in parentheses) induced by the large amplitude $e_{2}^{\prime \prime}$ deformation according to the POS normal coordinate treatment. All of these secondary displacements are symmetry-allowed as are others which appeared with smaller amplitudes. Previous assignments: L, Ref. 20; K, Ref. 22; C, Ref. 24; W, Ref. 26; E, Ref. 27; B, Ref. 29. 
TABLE II. Orbital energies (hartree) for $\mathrm{IF}_{7}$ with and without $d$ polarization functions. Two different conformations are included.

\begin{tabular}{|c|c|c|c|c|c|}
\hline \multicolumn{2}{|c|}{$C_{s}^{2}$ with $d^{b}$} & $D_{5 h}^{c}$ with $d$ & $D_{5 h}{ }^{\mathrm{c}}$ without $d$ & \multicolumn{2}{|l|}{$C_{s}^{2}$ without $d$} \\
\hline & -1.7188 & -1.7192 & -1.7041 & -1.7038 & $a^{\prime}$ \\
\hline & -1.7008 & -1.7012 & $-1.6778665 a_{2}^{\prime \prime}$ & -1.67733 & $a^{\prime}$ \\
\hline & -1.6473 & -1.6475 & $-1.619935 \quad a_{1}^{\prime}$ & -1.6197 & $a^{\prime}$ \\
\hline & $\left.\begin{array}{l}-1.6213619 \\
-1.6213610\end{array}\right\}$ & $-1.62125 \quad e_{1}^{\prime}$ & $-1.598847 \quad e_{1}^{\prime}$ & $\left\{\begin{array}{l}-1.5990296 \\
-1.5990291\end{array}\right.$ & $\begin{array}{l}a^{\prime \prime} \\
a^{\prime}\end{array}$ \\
\hline$a^{\prime}$ & -1.5924 & & & $\{-1.5594$ & $a^{\prime}$ \\
\hline$a^{\prime \prime}$ & -1.5923 & -1.59208 & $-1.5589284 e_{2}^{\prime}$ & $\{-1.5591$ & $a^{\prime \prime}$ \\
\hline$a^{\prime}$ & -1.0546 & -1.0546 & $-1.09670 \quad a_{\mathrm{i}}^{\prime}$ & -1.0967 & $a^{\prime}$ \\
\hline$a^{\prime}$ & -0.8747 & -0.87489 & $-0.8681987 a_{2}^{\prime \prime}$ & -0.8679 & $a^{\prime}$ \\
\hline$a^{\prime \prime}$ & $-0.8539505\}$ & $-0.8538963 e_{i}^{\prime}$ & $-0.8481847 e_{i}^{\prime}$ & $\{-0.8481483$ & $a^{\prime \prime}$ \\
\hline & $-0.8539504\}$ & $-0.8538963 e_{1}$ & -0.84818 & $1-0.8481481$ & $a^{\prime}$ \\
\hline & $-0.7983058\}$ & $-0.7982526 e_{1}^{\prime \prime}$ & $-0.7361888 e_{1}^{\prime \prime}$ & $\{-0.736117$ & $a^{\prime}$ \\
\hline & $-0.7983031\}$ & $-0.1000040 E_{1}$ & & -0.736113 & $a^{\prime \prime}$ \\
\hline & $\left.\begin{array}{l}-0.7695 \\
-0.7689\end{array}\right\}$ & $-0.7695656 e_{2}^{\prime}$ & $-0.7141876 e_{2}^{\prime}$ & $\left\{\begin{array}{l}-0.7141 \\
-0.7136\end{array}\right.$ & $\begin{array}{l}a^{\prime} \\
a^{\prime \prime}\end{array}$ \\
\hline$a^{\prime}$ & -0.7240 & $-0.72394 \quad a_{1}^{\prime}$ & -0.69421 & -0.6940755 & $a^{\prime}$ \\
\hline$a^{\prime \prime}$ & $-0.7156013\}$ & $-0.7156848 e_{1}^{\prime}$ & -0.0021201 & -0.6940723 & $a^{\prime \prime}$ \\
\hline & $-0.7155926\}$ & 2. & $-0.6450977 a_{2}^{\prime \prime}$ & -0.6448 & $a^{\prime}$ \\
\hline $\begin{array}{l}a^{\prime} \\
a^{\prime \prime}\end{array}$ & $\left.\begin{array}{l}-0.6701 \\
-0.6700\end{array}\right\}$ & $-0.6704705 e_{1}^{\prime \prime}$ & $-0.6408039 e_{1}^{\prime \prime}$ & $\left\{\begin{array}{l}-0.6403699 \\
-0.6403624\end{array}\right.$ & $\begin{array}{l}a^{\prime \prime} \\
a^{\prime}\end{array}$ \\
\hline$a^{\prime}$ & -0.6669 & $-0.66716 \quad a_{2}^{\prime \prime}$ & & $\{-0.6325$ & $a^{\prime}$ \\
\hline$a^{\prime}$ & $-0.6524053\}$ & $-0.6521902 e_{2}^{\prime \prime}$ & $-0.0308092 e_{2}$ & $\{-0.6310$ & $a^{\prime \prime}$ \\
\hline $\begin{array}{l}a^{\prime \prime} \\
a^{\prime}\end{array}$ & $-0.6523735)$ & & $-0.622139 \quad a_{1}^{\prime}$ & -0.620687 & $\begin{array}{l}a^{\prime} \\
a^{\prime \prime}\end{array}$ \\
\hline $\begin{array}{l}a \\
a^{\prime \prime}\end{array}$ & $\left.\begin{array}{l}-0.6272904 \\
-0.6272948\end{array}\right\}$ & $-0.6271937 e_{1}^{\prime}$ & $-0.5969851 e_{1}^{\prime}$ & $\left\{\begin{array}{l}-0.5972671 \\
-0.5972631\end{array}\right.$ & $a^{\prime}$ \\
\hline$a^{\prime}$ & -0.6018 & $-0.6106706 e_{2}^{f}$ & $-0.5647467 a_{2}^{\prime}$ & -0.565648 & $a^{\prime \prime}$ \\
\hline $\begin{array}{l}a^{\prime} \\
a^{\prime}\end{array}$ & $\begin{array}{l}-0.6107 \\
-0.5998^{d}\end{array}$ & $-0.59902^{\mathrm{d}} \quad a_{2}^{\prime}$ & $-0.5507735^{\mathrm{d}} e_{2}^{\prime}$ & $\left\{\begin{array}{l}-0.5511 \\
-0.5509^{d}\end{array}\right.$ & $\begin{array}{l}a^{\prime} \\
a^{\prime \prime}\end{array}$ \\
\hline$a^{r}$ & $0.0762^{\circ}$ & $0.07629^{\circ} \quad a_{1}^{\prime}$ & $0.0417196^{\circ} a_{1}^{\prime}$ & $0.0416^{\circ}$ & $a^{\prime}$ \\
\hline & 0.2072 & $0.2070459 a_{2}^{\prime \prime}$ & $0.1521260 a_{2}^{\prime \prime}$ & 0.1530 & $a^{\prime}$ \\
\hline & 0.2438 & $0.2465616 e^{\prime}$ & $0,1902487 e_{i}^{\prime}$ & $\{0.1896118$ & $a^{\prime}$ \\
\hline$a^{\prime \prime}$ & 0.2439 & & & $\{0.1896119$ & $a^{\prime \prime}$ \\
\hline $\begin{array}{l}a^{\prime} \\
a^{\prime \prime}\end{array}$ & $\left.\begin{array}{l}0.3575678 \\
0.3575688\end{array}\right\}$ & $0.3546422 e_{1}^{\prime \prime}$ & & & \\
\hline $\begin{array}{l}a \\
a^{\prime}\end{array}$ & $\begin{array}{l}0.3575688) \\
0.6480\end{array}$ & & & & \\
\hline$a^{\prime \prime}$ & $0.6483\}$ & $0.6478096 e_{2}^{\prime}$ & & & \\
\hline & 1.0363219 & $1.0388639 a_{1}^{\prime}$ & & & \\
\hline
\end{tabular}

${ }^{2} e_{2}^{\prime \prime}$ deformation from $D_{5 h}$, puckering amplitude of $3^{\circ}$ (see Ref. 16), with experimental bond lengths $\left(I-F_{2 J}\right)=1.786 \AA, r\left(I-F_{\text {eq }}\right)=1.858 \AA$.

Gaussian primitive with exponent 0.25 .

'Pentagonal bipyramid, bond lengths as in footnote a.

${ }^{\mathrm{d}}$ HOMO.

'LUMO.

\section{Extended Huckel force constants}

Extended Hückel computations of bending force constants were carried out with several different parametrizations, with and without $d$ orbitals on iodine, in order to assess the sensitivity of results to parameters. Parameters were shifted from neutral atom values ${ }^{36,40}$ to make allowance for the displacement of charge. Representative parameters were $-20.94,-15.61,-4.42$, -35.15 , and $-14.68 \mathrm{eV}$ for valence-orbital ionization potentials (VOIP) of $5 s, 5 p, 5 d, 2 s$, and $2 p$ Slater orbitals, respectively, whose orbital exponents were taken as $2.8,2.44,1.05,2.53$, and 2.52 .

Although absolute values of force constants were dependent upon the parameters, the pattern of bending force constants of interest here was not, as verified by calculations with different sets of orbital exponents and VOIP values.

\section{RESULTS}

In Table II are listed orbital energles for $\mathrm{IF}_{\eta}$, as calculated at $D_{5 h}$ and $C_{s}$ symmetries by our pseudopotential approach. For the benefit of those concerned with $d$ orbital involvement or with orbital mixings encountered in second-order Jahn-Teller inter actions, ${ }^{41-43}$ tabulations include results incorporating and excluding Gaussian $d$ orbitals on iodine. Force constants and bond lengths derived excluding $d$ orbitals are given in Table III. Bending force constants, as calculated by Hückel and VSE PR-POS calculations are demonstrated in Fig. 1 to be in semiquantitative agreement with the $a b$ initio force constants. Vibrational frequencies calculated from the pseudopotential force constants are tabulated in Table IV along with various spectroscopic results. Bending frequencies so calculated can be compared with observed frequencies ${ }^{24,27}$ according to prior published assignments ${ }^{20-29}$ and to our proposed assignment in Fig. 2. 
TABLE III. Calculated symmetry force constants (mdyn $/ \AA$ ), a cubic coupling constant (mdyn/ $\left.\AA^{2}\right)$, and bond lengths $(\AA)$ for $I F_{7}$ in $D_{5 h}$ configuration.

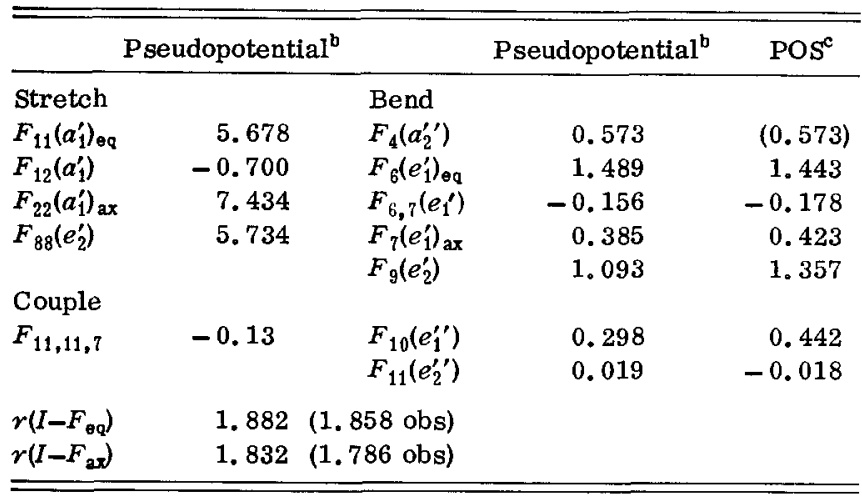

a Numbering convention of Khanna, Ref. 22. (1 mdyn $\AA$ $=10^{-18} \mathrm{~J}$.

${ }^{b}$ Taylor expansion about reference structure with experimental bond lengths, Ref. 16.

${ }^{c}$ Points-on-a-sphere model of Sec. II B using repulsion energy $\mathrm{Kr}^{-3.5}$ to reproduce observed ring pucker, Ref. 16, with constant $K$ adjusted to make $F_{4}$ fit the pseudopotential value.

Frequencies and mode-mixing implied by the POS field of Table I are also included in Fig. 2.

\section{DISCUSSION}

\section{A. Structure and anharmonic coupling of modes}

By all odds the most comprehensive analysis of $\mathbf{I F}_{7}$ has been carried out by Bernstein and Pitzer ${ }^{29}$ who took into account all of the evidence accumulated about struc ture, force field, and coupling with external fields prior to 1974 and fashioned a model to reproduce observations. This model, for mally embodying a second-order Jahn-Teller distortion ${ }^{41,42}$ considered to be induced by $e_{1}^{\prime}\left(F_{2 p}\right), e_{1}^{\prime \prime}\left(I_{5 d}\right)$ orbital mixing, was based on a molecular behavior closely patterned after that proposed to account for the electron diffraction intensities of $\mathrm{IF}_{7},{ }^{16}$ and $\operatorname{ReF}_{7 \cdot}{ }^{44}$

Experimental evidence ${ }^{16}$ indicates that $\mathrm{IF}_{7}$ in the vapor phase is a pentagonal bipyramid whose mean structure is distorted from $D_{5 h}$ symmetry by an $e_{2}^{\prime \prime}$ ring-puckering displacement of about $7.5^{\circ}$. Ring puckering, in turn, appears to induce a polar $e_{1}^{\prime}$ axial bend of perhaps $4.5^{\circ}$ via a cubic coupling constant $F_{11,11,7 \cdot}$ Equatorial bond lengths, averaging $1.858 \AA$, are $0.072 \AA$ longer than axial. All of the above features are easily understood in terms of the VSEPR theor $y^{10}$ according to which equatorial bonds, wedged into a much more congested environment than the axial bonds, tend to squeeze each other out-of-plane and expand. Note that the points-ona-sphere (POS) force constant $F_{11,11}\left(e_{2}^{\prime \prime}\right)$ of Sec. II B becomes negative when repulsions are sufficiently hard $\left(n>2\right.$ if $\left.V_{1} \propto r_{i j}^{-n}\right)$. A negative $e_{2}^{\prime \prime}$ force constant corresponds to a spontaneous ring-puckering displacement. The POS model even yields a coupling constant of the right magnitude to account for the observed correlation between $e_{2}^{\prime \prime}$ and $e_{1}^{\prime}$ deformations. ${ }^{16,29}$

A very similar picture of $\mathrm{IF}_{7}$ emerges from the pseudopotential calculations. These make the mole- cule a pentagonal bipyramid with equatorial bonds 0.05 $\AA$ longer than axial. Calculated bond lengths average $0.03 \AA$ longer than experimental, a systematic defect noted in the other fluorides of iodine and xenon treated in the present series of computations. Since all calculated quadratic force constants for displacement from $D_{5 h}$ symmetry turned out to be positive, the equilibrium structure implied is $D_{5 h}$, not a deformed bipyramid. It must be noted, however, that the calculated restoring force for $e_{2}^{\prime \prime}$ puckering is extremely weak, as shown in Table II. To appreciate how weak, it is instructive to consider the potential functions that would correspond to a simple diagonal valence force field. ${ }^{45}$ For this field the $e_{2}^{\prime \prime}$ force constant would be identical to the $e_{1}^{\prime \prime}, e_{1}^{\prime}$ (axial bend) and $a_{2}^{\prime \prime}$ constants. Therefore, it is apparent from Table II that the bond-bond interactions intrinsic in the present pseudopotential calculations cut down the $e_{2}^{\prime \prime}$ constant to a value 20 -fold below the level of others. That the calculation stopped just short of making the puckering constant negative is perhaps an accident of basis set or other factor of little significance. For that matter, not even experiment ${ }^{16}$ yielded a perfectly unequivocal conclusion about the equilibrium (minimum energy) structure. What is observed is the thermal distribution of structures. A molecule with a positive but very feeble restoring force would be seen as strongly puckered most of the time. Theory and experiment are in substantial agreement about the coupling between axial bend and pucker. Let us define the cubic constant $F_{11,11,7}$ by the equation

$V\left(e_{2}^{\prime \prime}, e_{1}^{\prime}\right)_{\mathrm{cubic}}=F_{11,11,7}\left[\left(S_{11 b}^{2}-S_{11 a}^{2}\right) S_{7 a}+2 S_{11 a} S_{11 b} S_{7 b}\right]$, adopting the symmetry coordinates of Fig. 3 of Ref. 44 and correcting the sign in Eq. (11), Chap. V, Ref. 29. Then the cubic constant calculated by the present pseudopotential method is $-0.13 \mathrm{mdyn} / \AA^{2}$, whereas the constant adjusted to fit the observed correlated displacements incorporated into the empirical field of Bernstein ${ }^{29}$ is $-0.19 \pm 0.07 \mathrm{mdyn} / \AA^{2}$.

If the combination bands assigned by vibrational spectroscopists ${ }^{20-29}$ in terms of $e_{2}^{\prime \prime}$ contributions of about $200 \mathrm{~cm}^{-1}$ have been correctly interpreted, then the equilibrium structure must be distorted from $D_{5 n}$. Only the "pseudoradial" vibrational component of a puckered pseudorotating molecule could have any chance of possessing that high a frequency for a nominally $e_{2}^{\prime \prime}$ mode, according to the present calculations. On the other hand, because existing assignments of combination bands are highly speculative, this argument has little force. The POS force field (Table I) suggests a pseudoradial $e_{2}^{\prime \prime}$ frequency well under $100 \mathrm{~cm}^{-1}$.

\section{B. Quadratic force field and assignments}

It is pleasing to find that $a b$ initio theory and experiment are in substantial agreement on structural aspects of $\mathrm{IF}_{7}$ and moreover, that both can be understood in terms of simple conceptual model (VSEPR) previous ly found to have wide validity in chemistry. ${ }^{9,10}$ No such agreement between experiment and conceptual model was apparent in the published force fields ${ }^{20-24}$ of $\mathrm{IF}_{7}$, how ever, when the structure was determined. The present work strongly suggests that the lack of agreement is not 
to be attributed to a failure of the conceptual model but rather to errors in the spectroscopic assignments. A somewhat analogous situation also arose for trigonal bipyramids. For a long period of time, spectroscopists found it plausible to assign higher bending force constants to shorter bonds. In this case, too, the error in assignments was detected when assignments were seen to be inconsistent with VSEPR theory. ${ }^{13}$

The profile of bending force constants implied by the VSEPR theory in its points-on-a-sphere representation is illustrated in Fig. 1(a) for various degrees of hardness of repulsion. Simple Coulomb potentials $(n=1)$ were never believed to be the source of the interactions and it is clear that they are too soft. An intermediate hardness, with $\mathrm{n}$ assigned the magnitude needed to account for the electron diffraction structure, is in surprising conformity with the $a b$ initio molecular orbital results in Fig. 1(c), as shown in Table III. Computations carried out by extended Hückel theory, of course, are utterly devoid of explicit Coulomb interactions, yet they also simulate the profile constructed from $a b$ initio theory. Despite the popular belief that $s$ and $p$ orbitals in a valence shell can support only four covalent bonds so that $d$ orbitals must be called into play to account for the seven bonds in $I F_{7}$, it can be seen in Fig. 1(b) that d orbitals play a minor role in the force field characteristics. This conclusion is in harmony with the $a b$ initio calculations comparing $D_{5 h}$ and $e_{2}^{\prime \prime}$ deformed structures, including and excluding $d$ orbitals (Table II), and the reasonable force field calculated without $d$ orbitals.

The weight of theoretical evidence as portrayed in Fig. 1, then, strongly implies that the stiff bending modes are the equatorial in-plane bends $\nu_{6}$ and $\nu_{9}$ for which lateral atomic motions are strongly impeded by the close in-plane contacts. This physical argument appears not to have guided the half-dozen published assignments $^{20,22,24,26,27,29}$ by spectroscopists, none of whom assigned what seems to be the highest frequency bend to an in-plane mode (see Fig. 2). Frequencies calculated by the present method are not expected to be quantitatively accurate but calculated bending frequencies for the similar molecules $\mathrm{XeF}_{2}$ and $\mathrm{XeF}_{4}$ were mostly within about $10 \%$ of the observed values. If it can be assumed that the present pseudopotential results are not off by much more than this and that their relative values are approximately correct, there is only one bending assignment which looks plausible, namely that indicated in Fig. 2. It agrees with the assignment of Bernstein and Pitzer $^{29}$ in all but one bending mode, the highly congested $e_{2}^{\prime}$ mode, but disagrees considerably from the others, all of which disagree with each other.

There is one troublesome feature of the present suggested assignment. It disregards as a separate mode the feature reported to be at $352 \mathrm{~cm}^{-1}$ by Claassen $e t$ $a l .{ }^{24}$ This not very sharp band of modest intensity could easily be taken to have a somewhat higher frequency, espectally in curve $B$ of Claassen's Fig. 1, depending upon the assumed slope of the background due to the broad $310 \mathrm{~cm}^{-1}$ band. If our suggested assignment is correct it is necessary to ascribe this band to the $363-$
$365 \mathrm{~cm}^{-1}$ mode of nominally $a_{2}^{\prime \prime}$ symmetry. Our nor mal coordinate calculations (POS) with a $C_{s}\left(e_{2}^{\prime \prime}+e_{1}^{\prime}\right)$ structure indicated a significant mixing in of an $e_{2}^{\prime}$ contribution (notation based on $D_{5 \hbar}$ point group) and, hence, a possibly significant Raman activity. Analogously, as can be seen in Fig. 2, there is a contribution of an infrared-active component in the $510 \mathrm{~cm}^{-1}$ Raman mode. Claassen's published infrared spectrum exhibits a feature possibly ascribable to this mixing.

If the $352 \mathrm{~cm}^{-1}$ feature is accounted for this way and the $510 \mathrm{~cm}^{-1}$ band is assigned as a bend, only four recognized bands remain, two in the infrared and two in the Raman spectra, to be distributed among the five stretching frequencies expected. There seems to be no doubt that Eysel's $a_{1}^{\prime}$ assignments ${ }^{27}\left[\nu_{1}(\mathrm{eq}) \approx 630 \mathrm{~cm}^{-1}\right.$, $\left.\nu_{2}(\mathrm{ax}) \approx 675 \mathrm{~cm}^{-1}\right]$ are correct for the two strong Ramanactive modes, because our relative pseudopotential axial and equatorial stretching force constants are not only nicely consistent with their assignments but also with Badger's rule $e^{16,46}$ and the observed bond lengths. The latter, in turn, with $r_{\mathrm{ax}}<r_{\mathrm{w}}$, are in accord with the simple VSEPR-POS picture. No new information about the infrared stretches was generated in the present study but there seems no reason to alter the more recent as signments. ${ }^{24-29}$

What should be done with the remaining $e_{2}^{\prime}$ Raman-active stretch $\nu_{8}$ can only be conjectured. Stretching frequencies calculated by the present pseudopotential method are systematically too high by perhaps $20 \%$ and relatively less reliable than bending frequencies. Our computations suggest that the $e_{2}^{\prime}$ stretch is not greatly different in frequency from the $a_{1}^{\prime}$ equatorial stretch. It is possible, then, that $\nu_{8}$ is either the weak $598 \mathrm{~cm}^{-1}$ feature in the foot of the $635 \mathrm{~cm}^{-1}$ band depicted by Claassen et al. ${ }^{24}$ in their Fig. 1, but not identified, or that it is buried under the $635 \mathrm{~cm}^{-1}$ band itself. In either case, it would have to be a very weak band. Whether this is reasonable is not answered by the present calculations.

Combination bands offer little guide in the resolution of assignments. Each of the six different assignments referred to in Table IV was considered to offer a satisfactory account of combination bands. Our assignments closely resemble those of Bernstein and Pitzer, ${ }^{29}$ differing principally in reassigning the $510 \mathrm{~cm}^{-1}$ band from an $e_{2}^{\prime}$ stretch to an $e_{2}^{\prime}$ bend. Accordingly, symmetry assignments are unchanged as are selection rules for combination bands. Some relaxation of $D_{5 h}$ selection rules can be expected anyway, because of the large amplitude $e_{2}^{\prime \prime}, e_{1}^{\prime}$ vibrations.

\section{Nature of POS interactions}

Whether the "repulsions" in the successful POS calculation of bending force constants are better thought of as due to atom-atom nonbonded interactions or valenceshell-electron-pair interactions mediated by the spoiling of, say, optimum covalent binding by deformations, can be answered plausibly. Quite reasonable atomatom interaction energies to be expected for $F \cdot \cdots F$ pairs were derived in paper I of this series. ${ }^{32}$ Bending force constants computed with the assumption that 
TABLE IV. Vibrational assignments and frequencies $\left(\mathrm{cm}^{-1}\right)$ for $\mathrm{IF}_{7^{*}}$

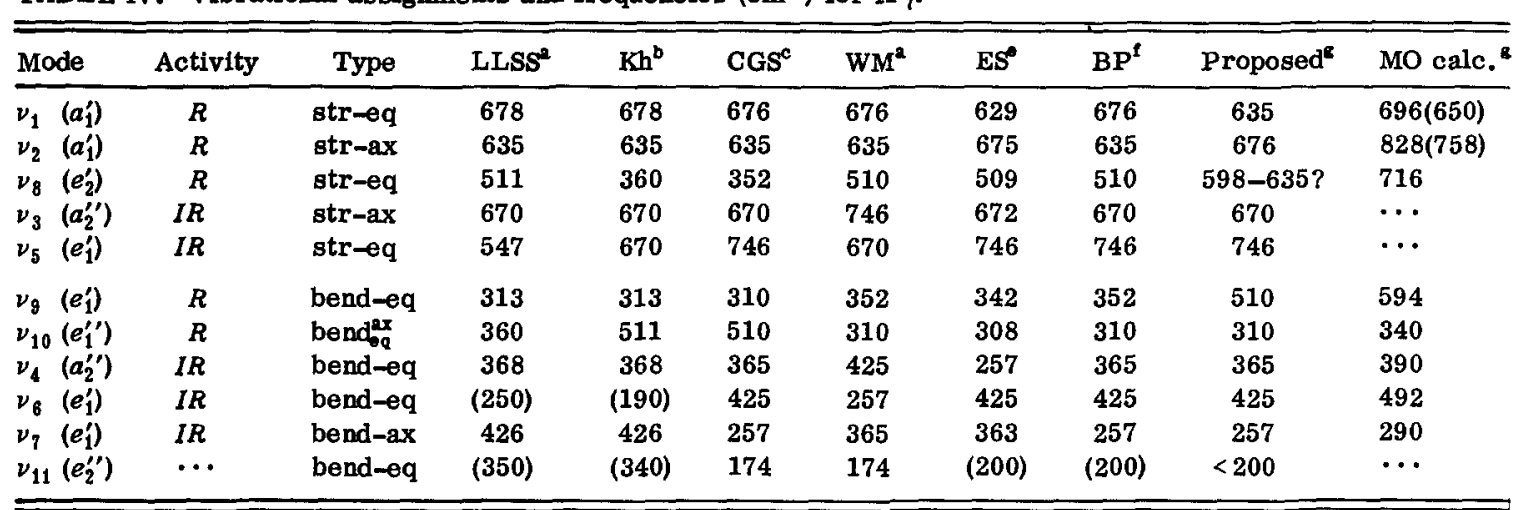

${ }^{2}$ Lord, Lynch, Schumb, and Slowinski, Ref. 20.

'Khanna, Ref. 22.

'Claassen, Gasner, and Selig, Ref. 24.

Wendling and Mahmoudi, Ref. 26

Eysel and Seppelt, Ref. 27.

${ }^{\mathrm{t}}$ Bernstein and Pitzer, Ref. 29.

${ }^{8}$ This work. Calculated frequencies used $F_{i j}$ computed at experimental bond lengths and (in parentheses) at calculated energy minimum; $G$ matrix of Khanna as corrected by Claassen and Kim.

atom-atom interactions are solely responsible for the deformation energies had the correct profile (like those in Fig. 1) but numerical values were severalfold too low.

\section{Comments on second-order Jahn-Teller effects}

In simple cases, the hierarchy of interactions implied by the Jahn-Teller expansion ${ }^{41,47}$ can offter insights into the structure or dynamics of molecules. ${ }^{41-43,47} \mathrm{~A}$ dozen years ago ${ }^{42}$ connections were pointed out between second-order Jahn-Teller effects (then more commonly called pseudo-Jahn-Teller effects $)^{48}$ and the valenceshell-electron-pair-repulsion (VSE PR) theory found to work so admirably in the present case of $\mathrm{IF}_{\mathbf{7}}$. In fact, the second-order Jahn-Teller effect was early invoked ${ }^{49,50}$ to account for the structure and dynamics of $\mathrm{XeF}_{6}$ which, in VSEPR theory, is formally similar to $\mathrm{IF}_{\mathrm{q}}$ in that both possess seven stereochemically active va-.. lence-shell electron pairs. Second-order Jahn-Teller theory is helpful when the energetics of deformation from a symmetric molecular configuration is dominated by the mixing of a single high-lying filled MO with an empty low-lying orbital. Many cases, including $\mathrm{XeF}_{6}$, have been examined in which this dominance appears to hold. In the present case, EHT calculations made the $e_{2}^{\prime \prime}$ deformation from $D_{5 h}$ symmetry spontaneous while the $a b$ initio pseudopotential calculations, instead, made the $e_{2}^{\prime \prime}$ deformations extremely soft, but not spontaneous. In neither set of calculations were the energetics of deformation dominated by a single orbital interaction; instead, contributions came from many symmetry-allowed interactions including some from rather low-lying valence orbitals. Therefore, there is little enlightenment to be derived from application of the second-order Jahn-Teller formalism in the present considerations.

As mentioned in the for egoing, Bernstein and Pitzer ${ }^{29}$ based their analysis of existing observations of $I F_{7}$ upon an $e_{2}^{\prime \prime}$ spontaneous deformation formally assumed to result from $e_{1}^{\prime}\left(F_{2 b}\right), e_{1}^{\prime \prime}\left(I_{5 d}\right)$ mixing. Our calculations show that this interpretation grossly exaggerates such mixing, for the lion's share of the softening of the $e_{2}^{\prime \prime}$ force constants is reproduced by calculations deleting $I_{5 d}$ orbitals. Moreover, the inclusion of $5 d$ orbitals in one set of computations did not lead to an increased softening. We wish to emphasize, however, that although our conclusion about the minor importance of the $F_{2 p}, I_{5 d}$ interactions conflicts with the formal basis of the Bernstein-Pitzer model, it only slightly impairs the practical utility of the molecular parameters derived by Bernstein. Only the magnitudes of the $e_{2}^{\prime \prime}$ potential constants are expected to be influenced appreciably.

\section{E. Remarks about the assignments of $\mathrm{ReF}_{7}$}

According to electron diffraction evidence ${ }^{16,44}$ the molecular structure and intramolecular motions of $\operatorname{ReF}_{7}$ closely resemble those $\mathrm{IF}_{7}$, except that the amplitudes of vibration in $\mathrm{ReF}_{7}$ are larger. It is reasonable to expect the pattern of bending force constants to resemble that of $\mathrm{IF}_{\eta}$, although there is some indication that bonds of transition metals, with a substantial involvement of $d$ orbitals, are less likely to be well-modeled by the POS approach than those of main-group elements. ${ }^{11}$ It is reasonable to re-examine the spectral assignments for ReF, by Claassen et al. ${ }^{24}$ with a view to trying to satisfy the POS implications. Presumably, we should expect the in-plane bending frequencies, topped by $e_{2}^{\prime}$, to be the highest. In the case of $\operatorname{ReF}_{7}$, indeed, these expectations are met by Claassen's assignments, even if they were not for $\mathrm{IF}_{7}$. We can find nothing to ques tion in his $\mathrm{ReF}_{7}$ assignments except, perhaps, for his low-frequency Raman bands. The $352 \mathrm{~cm}^{-1}$ band, tentatively assigned to $\nu_{10}\left(e_{1}^{\prime \prime}\right)$, may possibly be $\nu_{4}$ (nominally $a_{2}^{\prime \prime}$ rendered Raman active by $e_{2}^{\prime \prime}+e_{1}^{\prime}$ distortions, cf. the $352 \mathrm{~cm}^{-1}$ observed $\nu_{4}$ infrared frequency); the actual $\nu_{10}$ band may be in the low-frequency tail of this spectral feature, perhaps at the $281 \mathrm{~cm}^{-1}$ position labeled with a question mark by Claassen et al. If the present reassignments for $\mathrm{IF}_{7}$ are correct, the spectra of $\mathrm{IF}_{7}$ and $\operatorname{ReF}_{7}$, which were considered to be "strikingly different" by Claassen et al., can be seen to be less dissimilar than they originally appeared to be. 


\section{CONCLUDING REMARKS}

The present pseudopotential computations proved to be of value in several respects, even if the quantitative accuracy achieved was modest. They showed the feas ibility of exploring many facets of the potential surface of a rather complex molecule with a large number of electrons in a case where a comparable all-electron treatment would have been prohibitive. Prior computations ${ }^{30-34}$ with simpler, better understood molecules had delineated what systematic inaccuracies could be expected. The present results corresponded so closely to an interpretation of molecular behavior of $\mathrm{IF}_{7}$ deduced somewhat speculatively from electron diffraction data and reinfored by an analysis by Bernstein and Ptizer $^{29}$ that there now seems to be little doubt about the principal features of the molecule. Despite the limited quantitative accuracy of the potential constants derived, the results compellingly suggested errors in all of the half-dozen published assignments of vibrations, and pointed to an alternative assignment. The computations leading to these conclusions revealed an appealingly simple molecular physics governing molecular motions. Force constants deduced followed sur prisingly closely the patterns characteristic of a model of repelling points-on-a-sphere. It would appear advantageous in future studies of force fields to take greater advantage of this hint about molecular properties. Whether such a close correspondence between molecular orbital and points-on-a-sphere potential surfaces will survive in relativistic, multiconfiguration calculations remains to be seen.

Finally, the present results show that hypervalent compounds such as $\mathrm{IF}_{7}$ can be understood quite well without invoking valence $d$ orbitals on the central atom. These outer $d$ orbitals are neither the preponderant source of second-order Jahn-Teller softening of the ring-puckering force constant nor the sine qua non of covalent binding of seven ligands by a central atom with but four valence $s$ and $p$ orbitals.

\section{ACKNOWLEDGMENTS}

This research was supported by a grant from the National Science Foundation. We thank Professor C. S. Ewig and Professor J. R. Van Wazer for valuable advice and for the use of their pseudopotential program, and $\mathrm{Mr}$. V. Plato for his contributions to the extended Hückel calculations. We gratefully acknowledge computing time made available by the University of Michigan Computing Center.

${ }^{1}$ O. Ruff and R. Keim, Z. Anorg. Chem. 201, 245 (1931); American Institute of Physics Handbook, 2nd ed., edited by M. W. Zemanski (McGraw-Hill, New York, 1963), Sec. 4, pp. 4-222.

${ }^{2} \mathrm{~N}$. V. Sidgwick, The Chemical Elements and their Compounds (Oxford University, London, 1950), Vol. II, Chap. VII B.

${ }^{3}$ R. D. Burbank and F. N. Bensey, Jr., J. Chem. Phys. 27, 981 (1957).

${ }^{4} \mathrm{~J}$. Donohue, J. Chem. Phys. 30, 1618 (1959).

${ }^{5}$ R. D. Burbank, J. Chem. Phys. 30, 1619 (1959).

${ }^{6}$ L. L. Lohr, Jr. and W. N. Lipscomb, J. Chem. Phys, 36, 2225 (1962).

'R. D. Burbank, Acta Crystallogr. 15, 1207 (1962).
${ }^{8} \mathrm{~J}$. Donohue, Acta Crystallogr. 18, 1018 (1965).

N. V. Sidgwick and H. M. Powell, Proc. R. Soc. London, Ser. A 176, 153 (1940).

${ }^{10}$ R. J. Gillespie and R. S. Nyholm, Q. Rev. (London) 4, 339 (1957); R. J. Gillespie, Molecular Geometry (Van Nostrand, London, 1972).

${ }^{11}$ L. S. Bartell and V. Plato, J. Am. Chem. Soc. 95, 3097 (1973).

${ }^{12}$ L. S. Bartell, Kémiai Közlemenyek 48, 497 (1975).

${ }^{13}$ L. S. Bartell, Inorg. Chem. 9, 1594 (1970).

${ }^{14} \mathrm{H}$. B. Thompson and L. S. Bartell, Inorg. Chem. 7, 488(1968).

${ }^{15}$ T. A. Claxton and G. C. Benson, Can. J. Chem. 44, 157 (1966), and work cited therein.

${ }^{16} \mathrm{~W}$. J. Adams, H. B. Thompson, and L. S. Bartell, J. Chem. Phys. 53, 4040 (1970).

${ }^{17}$ Earlier, less complete work is reported by H. B. Thompson and L. S. Bartell, Trans. Am. Cryst. Soc. 2, 190 (1966); R. E. LaVilla and S. H. Bauer, J. Chem. Phys. 33, 182 (1960).

${ }^{18}$ E. W. Kaiser, J. S. Muenter, W. Klemperer, and W. E. Falconer, J. Chem. Phys. 53, 53 (1970).

${ }^{19}$ L. S. Bartell, J. Chem. Phys. 63, 3750 (1975); B. R. Miller and L. S. Bartell, ibid. 72, 800 (1980).

${ }^{20} \mathrm{R}$. C. Lord, M. A. Lynch, W. C. Schumb, and E. J. Slowinski, J. Am. Chem. Soc. 72, 522 (1950).

${ }^{21}$ G. Nagarajan, Curr. Sci. (India) 30, 413 (1961).

${ }^{22}$ R. K. Khanna, J. Mol. Spectrosc. 8, 134 (1962).

${ }^{23}$ L. S. Arighi, Ph. D. thesis, University of Wisconsin, 1965.

${ }^{24}$ H. H. Claassen, E. L. Gasner, and H. Selig, J. Chem. Phys. 49, 1803 (1968).

${ }^{25} \mathrm{~K}$. Ramaswamy and P. Muthusubramanian, J. Mol. Struct. 6, 205 (1970).

${ }^{26} \mathrm{E}$. Wendling and S. Mahmoudi, Bull. Soc. Chim. 1972, 33.

${ }^{27}$ H. H. Eysel and K. Seppelt, J. Chem. Phys. 56, 5081 (1972).

${ }^{28} \mathrm{~S}$. Mohan, Acta Cient. Indica 1, 31 (1978).

${ }^{29}$ L. S. Bernstein, Ph. D. thesis, University of California, 1974. Note that the number 4 in the numerator of the second term, left-hand side of Eq. [20(a)], p. 97, should be deleted.

${ }^{30}$ C. S. Ewig and J. R. Van Wazer, J. Chem. Phys. 63, 4035 (1975); C. S. Ewig, R. Osman, and J. R. Van Wazer, ibid. 66, 3557 (1977).

${ }^{31}$ M. J. Rothman, L. S. Bartell, C. S. Ewig, and J. R. Van Wazer, J. Comp. Chem. 1, 64 (1980).

${ }^{32}$ L. S. Bartell, M. J. Rothman, C. S. Ewig, and J. R. Van Wazer, J. Chem. Phys. 73, 367 (1980).

${ }^{33}$ M. J. Rothman, L. S. Bartell, C. S. Ewig, and J. R. Van Wazer, J. Chem. Phys. 73, 375 (1980).

${ }^{34}$ L. S. Bartell and A. Gavezzotti, Theochem. (in press).

${ }^{35}$ R. Hoffmann, J. Chem. Phys. 39, 1397 (1963).

${ }^{36}$ E. Clementi, D. L. Raimondi, and W. P. Reinhardt, J. Chem. Phys. 47, 1300 (1967).

${ }^{37} \mathrm{C}$. Roetti and E. Clementi, J. Chem. Phys. 60, 4725 (1974).

${ }^{38}$ Via a versatile program due to R. L. Hilderbrandt, Comput. Chem. 1, 179 (1979).

${ }^{39} \mathrm{~L}$. S. Bartell (unpublished research, 1972).

${ }^{40} \mathrm{H}$. Basch, A. Viste, and H. B. Gray, Theor. Chim. Acta 3, 458 (1965).

${ }^{41}$ R. F. W. Bader, Mol. Phys, 3, 137 (1960).

${ }^{42}$ L. S. Bartell, J. Chem. Educ. 45, 754 (1968).

${ }^{43}$ R. G. Pearson, J. Am. Chem. Soc. 91, 4947 (1969).

${ }^{44}$ E. J. Jacob and L. S. Bartell, J. Chem. Phys. 53, 2231 (1970).

${ }^{45}$ For example, the field of Ref. 22 which is diagonal in bending displacements.

${ }^{46}$ R. M. Badger, J. Chem. Phys. 2, 128 (1934); 3, 710 (1935).

${ }^{47}$ H. C. Lonquet-Higgins, Proc. R. Soc. London Ser. A 235, 537 (1956); U. Opik and M. H. L. Pryce, ibid. 238, 425 (1957).

${ }^{48}$ See J. Burdett, Chem. Soc. Rev. 7, 507 (1978).

${ }^{49}$ L. S. Bartell, J. Chem. Phys. 46, 4530 (1967).

${ }^{50}$ L. S. Bartell and R. M. Gavin, J. Chem. Phys. 48, 2466 (1968). 